\title{
Disfagia orofaríngea na criança: Condutas cirúrgicas
}

\author{
Rui Imamura ${ }^{1}$
}

\section{Resumo}

A disfagia orofaríngea, apesar de frequente nas unidades de terapia intensiva dos hospitais pediátricos, comumente gera dúvidas quanto às condutas mais adequadas para cada caso. Nesta aula apresentamos casos clínicos para ilustrar as condutas cirúrgicas mais utilizadas no manuseio destes pacientes, suas indicações, benefícios, limitações e complicações. Este conhecimento permite ao pediatra ponderar as alternativas de tratamento para melhora do estado nutricional e pulmonar de crianças com disfagia orofaríngea grave.

Palavras chave: disfagia orofaríngea; pneumonia aspirativa; tratamento cirúrgico

Imamura, Rui. "Disfagia orofaríngea na criança: Condutas cirúrgicas", in Anais do 2o. Congresso Internacional Sabará de Especialidades Pediátricas

\footnotetext{
${ }^{1}$ Doutor em Medicina pela Faculdade de Medicina da Universidade de São Paulo. Email: imamurar@terra.com.br
} 


\section{Introdução}

Através da discussão de casos clínicos, apresentamos as principais condutas cirúrgicas para tratamento de crianças com disfagia orofaríngea. Infelizmente, o nível de evidência cientifica disponível quanto à eficácia dos diversos tratamentos é baixo (Foley et al., 2008). Assim, a definição da conduta deve ser modelada para cada paciente, após ponderação dos potenciais benefícios, das limitações e riscos de cada procedimento e respeitando-se os desejos de pacientes e familiares.

\section{Injeção de toxina botulínica em glândulas salivares}

A toxina botulínica, injetada nas glândulas salivares, bloqueia o efeito da acetil-colina na sinapse neuro-glandular, inibindo a salivação. Como existem mediadores não colinérgicos da salivação, este bloqueio nunca é completo. Além disso, não é possível aplicar a toxina em todas as glândulas salivares. Como as glândulas submandibulares e parótidas são responsáveis pela maior parte da saliva produzida, elas são os principais alvos da aplicação. O uso da ultrassonografia auxilia a guiar a aplicação correta nos órgãos-alvo (Figura 1) e a limitar os efeitos deletérios da aplicação indevida em músculos da mastigação e da muculatura suprahióidea, importante para a fisiologia da deglutição. A duração do efeito é variável, sendo em média de três meses. Ensaios clínicos randomizados tem demonstrado a eficácia da toxina botulínica em diminuir a sialorréia, ou exteriorização da saliva, em diferentes populações (Chinnapongse et al. Mov Disord 2011; Wu et al. J Child Neurol 2011). Quando o objetivo é diminuir a aspiração de saliva e pneumonias aspirativas, a evidência é mais fraca, com algumas series de casos sugerindo redução do número de hospitalizações por pneumonia com uso da toxina (Raval e Elliott. Ann Otol Rhinol Laryngol 2008, Pena et al. J Vasc Interv Radiol 2009). 


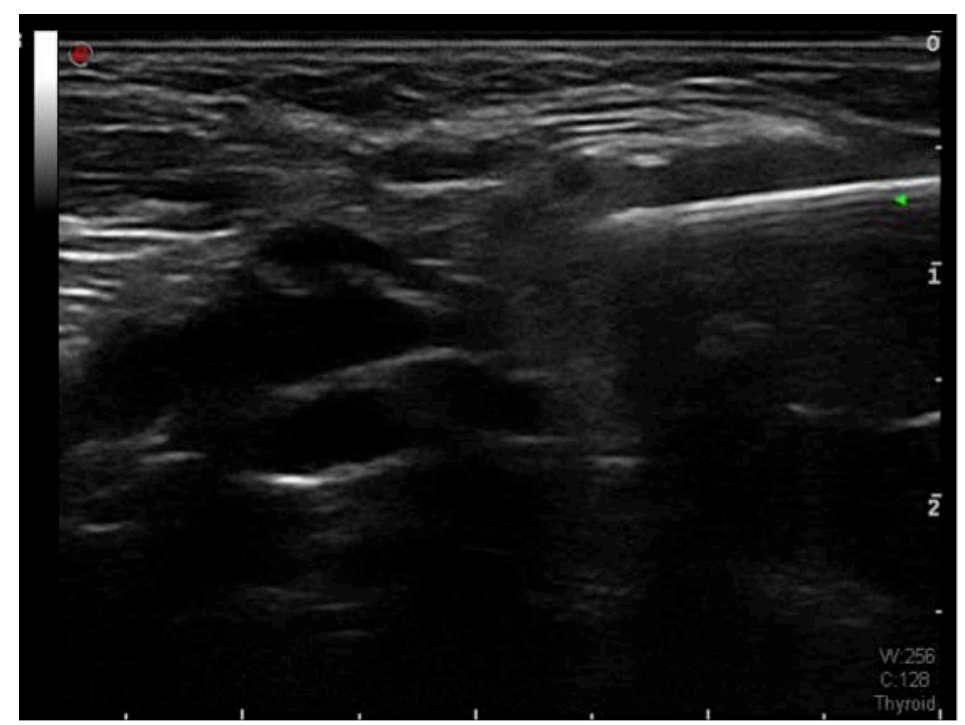

Figura 1: Ultrassonografia mostrando o posicionamento da agulha de injeção dentro da glândula salivar.

\section{Submandibulectomia e ligadura dos ductos parotídeos (Stensen) bilateral}

Conforme vimos, a injeção de toxina botulínica nas glândulas salivares pode auxiliar a diminuir tanto a sialorréia como a aspiração de saliva em crianças com disfagia orofaríngea. Contudo, seu efeito é temporário. Nas crianças em que o prognóstico de recuperação funcional da disfagia não é favorável, pode ser interessante obter uma redução da produção salivar mais definitiva. Nestes casos, a cirurgia para remover as glândulas submandibulares pode ser indicada (Figura 2). Como uma parotidectomia total implicaria em aumento da morbidade e tempo cirúrgico, a alternativa mais plausível é a ligadura bilateral dos ductos parotídeos (Figura 3). Apesar de promover uma retenção salivar e distensão glandular aguda após a cirurgia, tal procedimento acaba promovendo atrofia funcional da glândula, confirmada por evidências histológicas e radiológicas (Klem e Mair. Arch Otolaryngol Head Neck Surg, 1999). A cirurgia das glândulas salivares pode ter eficácia superior à injeção de toxina botulínica, uma vez que a injeção pode não atingir todo o parênquima glandular e, conforme citado, nem toda produção salivar é mediada por receptores colinérgicos. Segundo alguns autores, pode ter eficácia comparável à separação laringo-traqueal em pacientes com disfagia grave e aspiração de saliva (Gerber et al. Arch Otolaryngol Head Neck Surg, 1996). Por outro lado, a ligadura é plasível de fistulização pós-operatória, com perda do bloqueio salivar da respectiva glândula e eventual necessidade de reintervenção. 


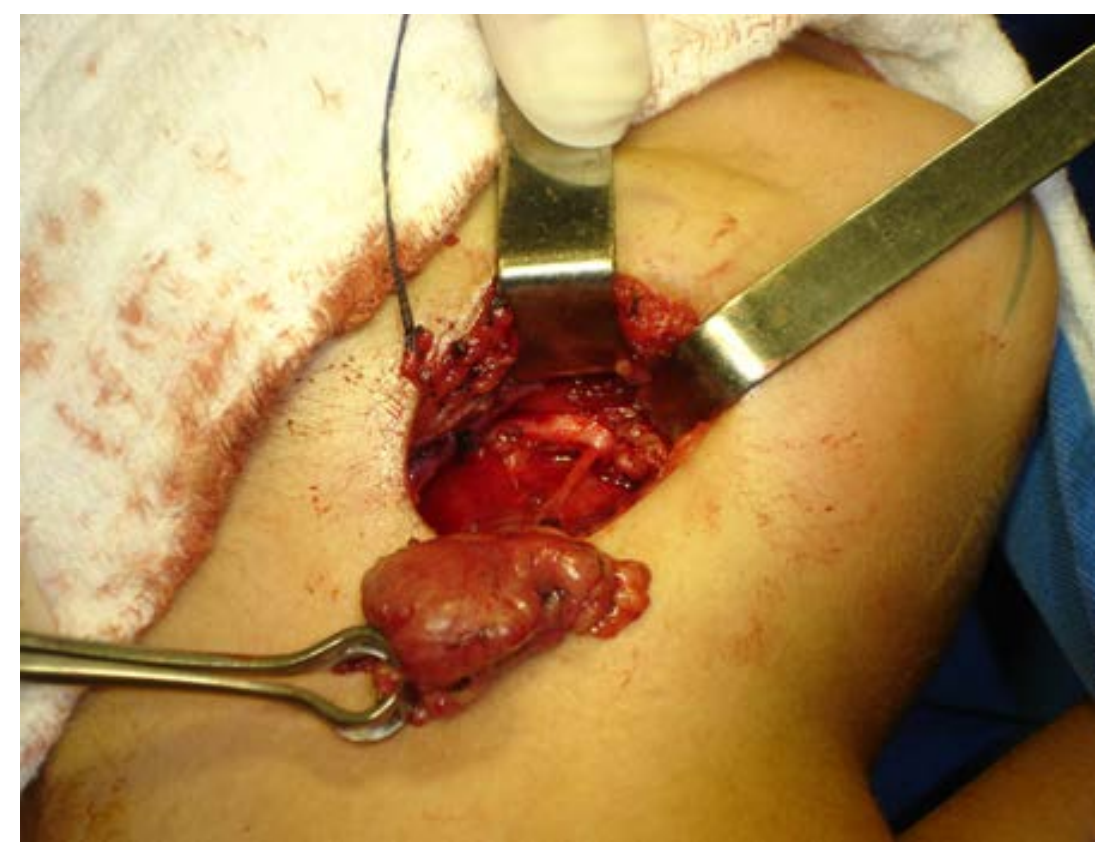

Figura 2: Submandibulectomia direita

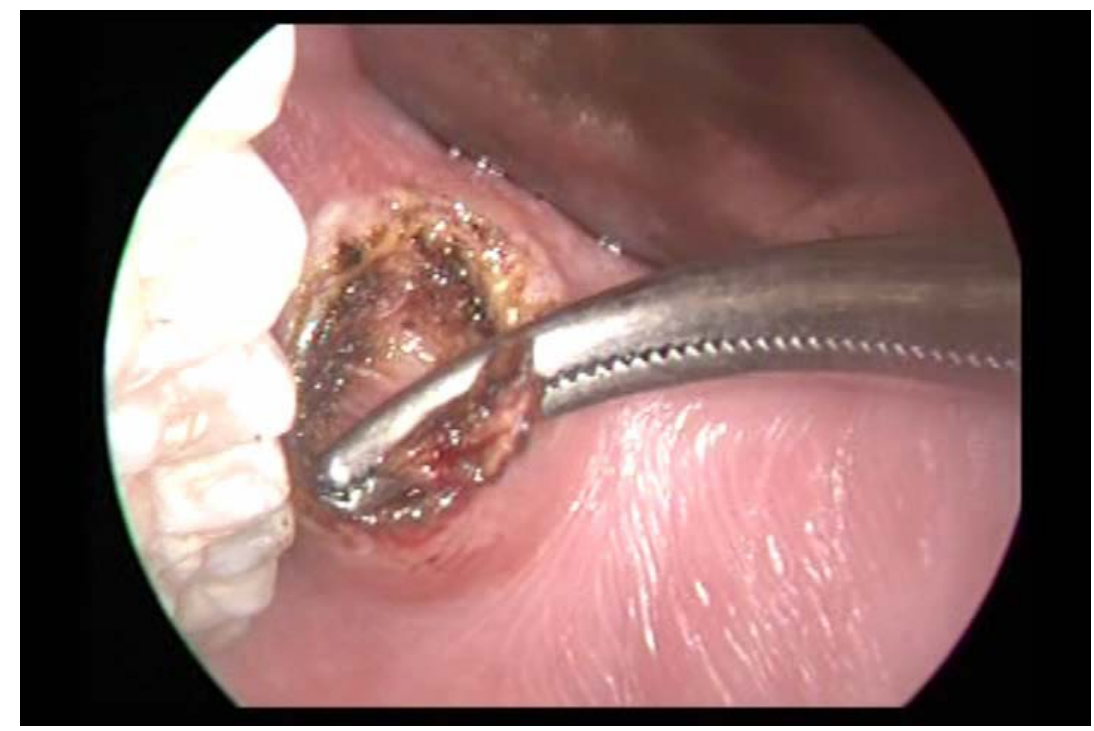

Figura 3: Ligadura do ducto parotídeo esquerdo

\section{Separação (ou desconexão) laringotraqueal}

Quando a aspiração salivar e as complicações pulmonares são graves, pode ser necessário adotar condutas extremas para controlar a aspiração. Em nosso entendimento, a cirurgia padrão-ouro para este fim é a separação laringotraqueal. Como a cirurgia separa a via aérea (pela confecção de um traqueostoma com a traquéia distal) da via digestiva, impede-se a aspiração de saliva. O coto proximal da traquéia é suturado em fundo-cego, em camadas, para evitar a fistulização. Apesar de eficaz para o controle da aspiração salivar, este procedimento elimina a vocalização e deste modo costuma encontrar resistência por parte de pacientes e familiares. Por outro lado, em pacientes já 
traqueostomizados e com vocalização limitada, em nossa experiência, existe pouca diferença em termos de morbidade, mas evidente melhora da condição pulmonar e clínica geral. Além de controlar as pneumonias aspirativas, alguns pacientes deixam de ser dependentes de oxigênio e passam até a se alimentar por via oral, apesar de não dispormos de fatores preditores para identificar tais pacientes. A cirurgia pode ser revertida em caso de melhora da disfagia, com reestabelecimento da via respiratória, possibilidade de fechamento do traqueostoma e recuperação funcional da voz.

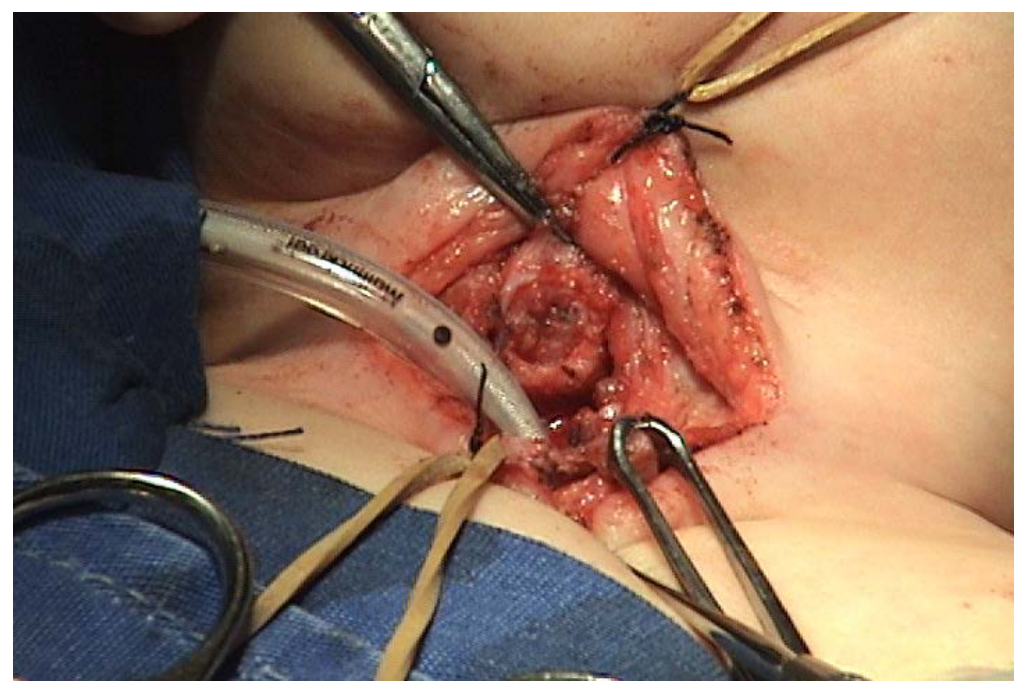

Figura 4: Separação laringotraqueal. O coto proximal da traqueia está suturado em fundo cego. A traqueia distal é suturada à pele, constituindo o traqueostoma.

\section{English Title}

Oropharyngeal dysphagia in children: Surgical treatment

\section{Abstract:}

Oropharyngeal dysphagia, although common in intensive care units in pediatric hospitals, commonly raises doubts as to the most appropriate approaches for each case. In this lecture, we present clinical cases to illustrate the surgical procedures most commonly used in these patients, their indications, benefits, limitations and complications. This knowledge allows the pediatrician to consider alternative treatments to improve the nutritional and pulmonary status of children with severe oropharyngeal dysphagia.

\section{Keywords:}

Oropharyngeal dysphagia; aspiration pneumonia; surgical treatment

\section{Referencias}

CHINNAPONGSE R, GULLO K, NEMETH P, ZHANG Y, GRIGGS L. Safety and efficacy of 
botulinum toxin type $B$ for treatment of sialorrhea in Parkinson's disease: a prospective double-blind trial. Mov Disord. 2012 Feb;27(2):219-26. doi: 10.1002/mds.23929. Epub 2011 Sep 1. PubMed PMID: 21887710.

FOLEY N, TEASELL R, SALTER K, KRUGER E, MARTINO R. Dysphagia treatment post stroke: a systematic review of randomised controlled trials. Age Ageing. 2008 May;37(3):258-64. doi: 10.1093/ageing/afn064. Review. PubMed PMID: 18456790.

GERBER ME, GAUGLER MD, MYER CM 3rd, COTTON RT. Chronic aspiration in children. When are bilateral submandibular gland excision and parotid duct ligation indicated? Arch Otolaryngol Head Neck Surg. 1996 Dec;122(12):1368-71. PubMed PMID: 8956752.

KLEM C, MAIR EA. Four-duct ligation: a simple and effective treatment for chronic aspiration from sialorrhea. Arch Otolaryngol Head Neck Surg. 1999 Jul;125(7):796-800. PubMed PMID: 10406320.

PENA AH, CAHILL AM, GONZALEZ L, BASKIN KM, KIM H, TOWBIN RB. Botulinum toxin A injection of salivary glands in children with drooling and chronic aspiration. J Vasc Interv Radiol. 2009 Mar;20(3):368-73. doi: 10.1016/j.jvir.2008.11.011. Epub 2009 Jan 20. PubMed PMID: 19157908.

RAVAL TH, ELLIOTT CA. Botulinum toxin injection to the salivary glands for the treatment of sialorrhea with chronic aspiration. Ann Otol Rhinol Laryngol. 2008 Feb;117(2):118-22. PubMed PMID: 18357835.

WU KP, KE JY, CHEN CY, CHEN CL, CHOU MY, PEI YC. Botulinum toxin type A on oral health in treating sialorrhea in children with cerebral palsy: a randomized, double-blind, placebo-controlled study. J Child Neurol. 2011 Jul;26(7):838-43. doi: 10.1177/0883073810395391. Epub 2011 May 6. PubMed PMID: 21551374. 